\title{
Alveolar Bone Loss, Plaque Index, and Gingival Index among Non-Diabetics Treated with Class II Amalgam Restorations for Different Service Lives
}

\author{
Mohammad Albakry* \\ Department of Dentistry, University of Najran, Kingdom of Saudi Arabia
}

*Corresponding author: Mohammad Albakry, Assistant Professor, Restorative Dental Sciences, Biomaterials, Faculty of Dentistry, University of Najran, PO Box 1988, Najran, Kingdom of Saudi Arabia.

To Cite This Article: Mohammad Albakry. Alveolar Bone Loss, Plaque Index, and Gingival Index among Non-Diabetics Treated with Class II Amalgam Restorations for Different Service Lives. Am J Biomed Sci \& Res. 2021 - 13(6). AJBSR.MS.ID.001924.

DOI: 10.34297/AJBSR.2021.13.001924.

Received: 眥 July 28, 2021; Published: 制 August 10, 2021

\begin{abstract}
Objective: The present study aimed to evaluate the influence of different service lives of class II amalgam restorations on periodontal health among patients without diabetes.

Materials and Methods: Two hundred and twenty-five patients without diabetes were divided into five groups (G1-G5) based on the ages of their class II amalgam restorations, that is, 2, 4, 7, 10, and > 10 years, respectively. Each group had 45 patients, aged $45-60$ years. They were assessed for the plaque index (PI), gingival index (GI), rate of overhangs $(\mathrm{OH})$, and alveolar bone loss (ABL), (OH and ABL, using panoramic radiographs). The data were analysed using the Scheffé test and analysis of variance.
\end{abstract}

Results: The periodontal parameters of the examined groups demonstrated low and intermediate scores that ranged from $25 \%$ to $41 \%$ for $\mathrm{ABL}$, 1.1 to 1.5 for PI, and 1.2 to 1.5 for GI. G4 and G5 had the highest scores for the three parameters (PI, GI, and ABL); $<<0.001$. The rate of overhangs varied among groups considerably with G1 and G3 having the highest rate $(\mathrm{p}<0.037)$.

Conclusion: The worse periodontal parameters among G4 and G5 indicated severe periodontal destruction among participants of the two groups, while the participants of G1, G2, and G3 had relatively better periodontal status indicated by their low and intermediate periodontal scores. Keywords: Class II amalgam restorations, Alveolar bone loss, Plaque index, Gingival index, Overhangs, Periodontitis, Periodontal health

Abbreviations: PI: Plaque Index; GI: Gingival Index; ABL: Alveolar Bone Loss; OH: C Overhanging Restorations; $\quad$ CEJ: Cementoenamel Junction

\section{Introduction}

Periodontal disease is a chronic immunoinflammatory multifactorial illness of the oral cavity occurring owing to bacterial deposition and may cause several periodontal complications, such as alveolar bone destruction [1-3]. The origin and transmission of the periodontal disease occur via dysbacteriosis of the commensal oral microbiota, which then interacts with the immune defence of the host, leading to inflammation and disease [4,5]. It is one of the most frequently diagnosed oral complaints in the human population [6]. An advanced form of periodontal disease, possibly leading to teeth loss, is highly common, with approximately $20 \%$ -
$50 \%$ of the global population affected $[7,8]$. In severe periodontitis, the gingiva can pull away from the tooth (recede) and supporting bone, resulting in bone resorption, causing loosening of teeth and sometimes exfoliation $[9,10]$.

Dental plaque is the primary causative factor for most periodontal diseases $[11,12]$. Many previous reports have addressed the essential role of dental plaque in periodontal diseases [13-15]. These reports demonstrated evidence of a definite correlation between plaque and periodontal diseases. The accumulation of plaque biofilm on the teeth and surrounding soft tissues is linked 
to a stage of inflammatory alteration in the oral tissues [16]. Such periodontal alteration can be quantified or diagnosed by examining basic clinical oral signs and assessing certain periodontal indices [17]. The most significant periodontal parameters that are routinely used to determine different stages of periodontal diseases are plaque index (PI), gingival index (GI), and alveolar bone loss (ABL).

The ongoing research has identified pertinent risk factors that play a significant role in the initiation and progression of periodontal inflammation. Many systemic and local factors are known to initiate and aggravate periodontal disease. A significant local factor is the quality of restorations performed in relation to the periodontal tissues. Faulty restorations are most likely to cause negative effects on the oral health status. This consistently present as an elucidation for the detrimental effects of restorations on periodontal status $[18,19]$. Certain essential restoration factors can negatively influence periodontal tissues, if not considered during restorative treatment. Some of these factors are 1) type of restoration, 2) marginal adaptation, 3) proximal relationships, 4) surface smoothness of the restoration, and 5) inflammation around the restored tooth. These factors not only dictate a relationship between restoration and the resultant periodontal diseases but also can play a significant role in the success and longer service life of a specific restoration [20].

A significant factor when designing proximal restorative treatment, such as class II restoration, is the overhanging margin. In addition to disrupting the dynamic relationship that exists between a tooth and periodontium, overhanging margin promotes plaque accumulation, via providing an expedient site for diseaseassociated harmful organisms, eventually leading to a remarkable change in the ecological balance of the gingival sulcus region [21]. Moreover, this can be aggravated by a longer service life (age) of restorations. Older overhung restorations may encourage further growth of microbial dental plaque. This not only results in pathogenic multiplication but also gives rise to further periodontal destruction and ABL [22]. Hence, periodontal complications along with $\mathrm{ABL}$, related to amalgam restoration overhang, were highly prevalent, as stated in previous research studies [22-25]. However, the size of the overhang and the affected site should also be relevant in this association. The most problematic restorations associated with massive ABL are those with larger overhangs, greater than 1 $\mathrm{mm}[22,23]$.

Many research studies were found in the literature that evaluates the effect of different restorative dental materials on periodontal health [20,26-30]. However, no studies were found that appraise the effect of different service life (age) of class II amalgam restoration on periodontal health. Therefore, this study aimed to evaluate the effect of different service lives of class II amalgam restoration on periodontal health among patients without diabetes. It was hypothesized that patients with older class II amalgam restorations would have worse periodontal parameters, PI, GI, and $\mathrm{ABL}$, than those with shorter restorations' ages.

\section{Materials and Methods}

This research project was approved by the Research and Ethics Committee of the Faculty of Dentistry, Najran University, Kingdom of Saudi Arabia. The research project approval number is $003 / 18$ (January 1, 2018). All clinical examinations and/or evaluations, performed in the current study, which involved human participants, were as per the ethical standards of the institutional and/or national research committee, and the Helsinki declaration, as amended by the $64^{\text {th }}$ WMA General Assembly, Fortaleza, Brazil, October 2013, and its later amendments or comparable ethical standards. Additionally, signed consent forms were obtained from all examined patients who participated in the current study, before commencing.

\section{Sample Size and Sampling}

The sample size was calculated using the formula that used for calculating sample size, in medical research, proposed by Crano et al, 2002 [31], as follows:

$$
\mathrm{n}=\mathrm{Nn} / \mathrm{N}+\mathrm{n}^{*}
$$

where $\mathrm{n}$ is the required sample size, $\mathrm{N}$ is the population size (total number of participants, 650), and $\mathrm{n}^{*}$ is the first estimated sample. The first estimated sample $\left(\mathrm{n}^{*}\right)$ was determined using the following formula: $\mathrm{n}^{*}=\mathrm{P}(1-\mathrm{P}) /(\mathrm{SE})^{2}$

Where $\mathrm{P}$ is the estimated proportion to participants, which was assumed as 0.5 for getting the maximum sample size. SE is the standard error, assumed as 0.05 . Therefore, the first estimated sample, $n^{*}=100$; consequently, the sample size (n) is 86.7. It should be noted that any further increment in the population size would have a limited effect on the results [31].

\section{Inclusion and Exclusion Criteria}

The inclusion and exclusion criteria for the selection of the participants adopted in the current study were as follows:

Inclusion criteria: The inclusion criteria included: (1) patient without diabetes, (HbA1c levels

$4 \%-5 \%$ ), (2) 45-60 years of age, (3) a minimum of 15 teeth remaining, and (4) with one or more class II amalgam restorations aged $\geq 2$ years. 
Exclusion criteria: The exclusion criteria included (1) selfreported medical chronic conditions, such as diabetes mellitus, human immunodeficiency virus, cardiovascular complications, hepatic disorders, renal disorders, or epilepsy, (2) a history of previous antibiotic use or steroid therapy in the past 3 weeks, (3) immunosuppressive chemotherapy, (4) periodontal treatment for the past 6 months, (5) edentulous patients, and (6) crowding teeth or occlusal trauma.

\section{Study Population}

Since this was an observational comparative cross-sectional study, the participants were clinically examined with their current dental status. Moreover, no dental treatment was provided to the participants before the clinical examination. Among a sample of 650 patients who presented at the specialized dental clinics, Faculty of Dentistry, Najran University, Kingdom of Saudi Arabia, 225 male patients without diabetes were selected. They were divided according to the age of class amalgam restorations into five groups, group 1 to group 5 (G1-G5). G1-G5 had their class II amalgam restorations for an approximate duration of the following service lives: 2, 4, 7, 10, and 12.5 years. Each group comprised 45 patients, aged 45-60 years. It is noteworthy that female participants were not included in the current study as the dentistry program of the current educational institution (University of Najran) is specified for male students only. Therefore, this study sample included only men.

\section{Clinical Examinations}

All participants were subjected to clinical oral examination including periodontal tissues status. The screening clinical examination included the state of periodontal tissues by assessing the amount of dental plaque, plaque index (PI), and gingival condition, and qualitative alterations of the gingiva, gingival index (GI) using the two scoring systems proposed by: 1) Silness and Löe, 1964, [32] for PI, and 2) Löe and Silness, 1963, [33] for GI. For PI evaluation, four sides per tooth (buccal, lingual, mesial, and distal) were examined for each participant (except for third molars), using William's periodontal probe. For GI assessment, certain teeth were chosen: $16,12,24,32,36$, and 44 .

Two periodontal investigators collected the periodontal parameters, PI and GI. Values obtained were compared, and the overall kappa score for intra-examiner reliability was calculated.

\section{Radiographical Procedure}

Panoramic radiography was used to measure $\mathrm{ABL}$, and to locate sites of overhanging restorations. A panoramic X-ray unit (Planmeca Promax, Dent-R100, RPX253665, Helsinki, Finland) was used to produce the necessary images. The desired resolution of each radiographical procedure was selected, followed by adjusting the height of the X-ray according to patient height. The patient's chin was placed in the chin cup and the occlusal plane was set horizontally. The patient was directed to grasp the patient's handles tighten head support. The laser position was adjusted to correspond with the illustration on the touch screen. Final and fine adjustments were performed to volume location whenever needed. All panoramic radiographs were digitized and analyzed using a computer-assisted system for linear measurements.

Panoramic radiographs and ABL measurements were recorded for all participants during regular daily dental practice visits between March 2018 and February 2020.

\section{Panoramic Radiographs}

Forty-five complete sets of panoramic radiographs were obtained from 45 participants of each of the examined groups, (G1-G5); the total number of all panoramic radiographs being 225 . The examined groups (G1-G5) should correspond to the following service life of class II amalgam restorations, $2,4,7,10$, and $>10$ years.

The mean ABL along with overhanging restorations of class II restorations, among treated posterior teeth were considered irrespective of their class (premolars or molars), site (upper or lower), or side (mesial or distal).

The criteria for the acceptability of the panoramic radiographs were: 1) clear visibility of the anatomical features, such as cementoenamel junction (CEJ), alveolar bone crest (ABC), and tooth apices (AP), 2) CEJs were not compromised by the presence of a restoration, prostheses, traversing images, or defective radiographic image, and 3) both proximal sites (mesial and distal) were measurable. At the end of the selection process for the set of images, radiographic images that did not fulfil the criteria were discarded. A computer screen was used to amplify and visualize images.

The distance between the CEJ and crest of the alveolar bone, and the crest of the alveolar bone and tooth apex, were used to identify the alveolar bone with bone loss. ABL was recorded when the distance between the CEJ and the $\mathrm{ABC}$ was $>2 \mathrm{~mm}$. The ABL was presented as percentage bone loss [34].

Bone loss percentage was calculated using the following formula [34]

$$
((\mathrm{CEJ}-\mathrm{ABC})-2 \mathrm{~mm}) /((\mathrm{CEJ}-\mathrm{AP})-2 \mathrm{~mm}) \times 100
$$

Two periodontal investigators collected all clinical data. The collected data were grouped on Excel spreadsheets to record the percentage of alveolar bone level, root length, and bone loss in $\mathrm{mm}$. The ABL assessments produced by the two investigators were compared, and the overall kappa score for intraexaminer reliability was calculated. 


\section{Statistical Analysis}

Data were evaluated statistically using IBM SPSS version 25 . The variables were expressed as mean \pm SD and analyzed using Scheffe test, and one-way analysis of variance (ANOVA). A p-value $<0.05$ was considered statistically significant and $p<0.0001$ was deemed extremely significant.

\section{Results}

The mean ages of participants of the examined groups G1-G5 were 53.5 (SD 4.3), 52 (SD 4.1), 54 (SD 4.4), 57.5 (SD 3.5), and 59 (2.7) years; (Table 1). No significant difference in the mean ages of the first three groups, G1-G3 ( $p=0.392$ ) was noted. The participants of G4 and G5 were significantly older than those of the other three groups, G1-G3, ( $\mathrm{p}=0.03)$, with no significant difference between them $(\mathrm{p}<0.001)$.

The mean ages of the class II amalgam restorations in the patients in G1-G5 were 2.1 (SD 0.2), 4.3 (SD 0.4), 7 (SD 0.7), 10.3 (SD 1.3), and 12.8 (SD 2) years (Table 2). Furthermore, statistical analysis showed that these service life durations significantly differed ( $p<0.001)$. Regarding the overhanging class II amalgam restorations, the chi-square test showed that class II amalgam restorations differed significantly among all groups ( $p<0.001)$. G1 had a significantly higher rate of overhanging restorations $(60 \%)$ than those of the other groups $(\mathrm{p}<0.001)$, whereas the lowest rate of overhanging restorations ( $40 \%$ ) was recorded in G5 ( $p<0.001)$. The overhangs' rates among the examined groups are listed in Table 1.

\begin{tabular}{|c|c|c|c|c|c|}
\hline Group & $\begin{array}{c}\text { Mean age of } \\
\text { participants (SD) } \\
Y\end{array}$ & $\begin{array}{l}\text { Total no. of class } \\
\text { II amalgam } \\
\text { restorations }\end{array}$ & $\begin{array}{c}\text { Mean age of } \\
\text { class II amalgam } \\
\text { restorations (SD) }\end{array}$ & $\begin{array}{l}\text { Total no. of } \\
\text { overhanging } \\
\text { restorations }\end{array}$ & $\begin{array}{c}\text { Prevalence of overhanging } \\
\text { restorations } \\
\%\end{array}$ \\
\hline G1 & $53.5(4.3)$ & 45 & $2.1(0.2)$ & 27 & 60 \\
\hline G2 & $52(4.1)$ & 45 & $4.3(0.4)$ & 22 & 48.9 \\
\hline G3 & $54(4.4)$ & 45 & $7(0.7)$ & 25 & 55.6 \\
\hline G4 & $57.5(3.5)$ & 45 & $10.3(1.3)$ & 20 & 44.5 \\
\hline G5 & $59(2.7)$ & 45 & $12.8(2)$ & 18 & 40 \\
\hline
\end{tabular}

Table 2: Mean plaque index, mean gingival index, and mean alveolar bone loss of the examined groups.

\begin{tabular}{|c|c|c|c|}
\hline Group & Mean plaque index (SD) & Mean gingival index (SD) & Mean alveolar bone loss (SD)\% \\
\hline G1 & $1.1(0.16)$ & $0.9(0.3)$ & $25(2.5)$ \\
\hline G2 & $1.2(0.13)$ & $1.1(0.16)$ & $27(4.2)$ \\
\hline G3 & $1.45(0.14)$ & $1.25(0.35)$ & $34(3.95)$ \\
\hline G4 & $1.75(0.16)$ & $1.5(0.3)$ & $38.5(4.2)$ \\
\hline G5 & $2(0.19)$ & $1.75(0.16)$ & $41(4.7)$ \\
\hline
\end{tabular}

The mean values of the PI and GI scores and the SD of all examined groups are listed in Table 2 . The mean values of these indices for G1-G5 were 1.1 and 0.9 (SD 0.16, 0.15), 1.2 and 1.1 (SD $0.13,0.16$ ), 1.45 and 1.25 (SD 0.14, 0.35), 1.75 and 1.5 (SD 0.16, 0.3 ), and 2 and 1.75 (SD 0.19, 0.16). No statistically significant difference in the two values was observed between G1 and G2 (p $=0.42)$. The two values of G3-G5 differed significantly $(p<0.001)$. Statistically, G5 had the highest two values of all groups ( $\mathrm{p}<0.001)$.

Table 2 lists the results of the ABL and the standard deviation for all examined groups. The mean percentages of the ABL $( \pm \mathrm{SD})$ recorded in G1-G5 were 25\% (SD 2.5\%), 27\% (SD 4.2\%), 34\% (SD $3.95 \%$ ), 38.5\% (SD 4.6\%), and 41\% (SD 4.7). ANOVA revealed that patients in G4 and G5 demonstrated the highest mean percentage of ABL among the examined groups $(\mathrm{p}<0.001)$, with no significant difference between them ( $p=0.329)$. The smallest amount of ABL among all examined groups was observed in G1 and G2, with no significant difference between them ( $p=0.987)$. G3 recoded significantly higher ABL values than those recorded by G1 and G2 $((\mathrm{p}<0.001)$. This was an intermediate value between the lowest values recorded by G1 and G2 and the highest values recorded by G4 and G5.

Comparison of the assessment of the ABL made by the two investigators produced an overall kappa score of 0.85 for intraexaminer reliability.

Representative ABL images for all examined groups are shown in Figures 1-4. 


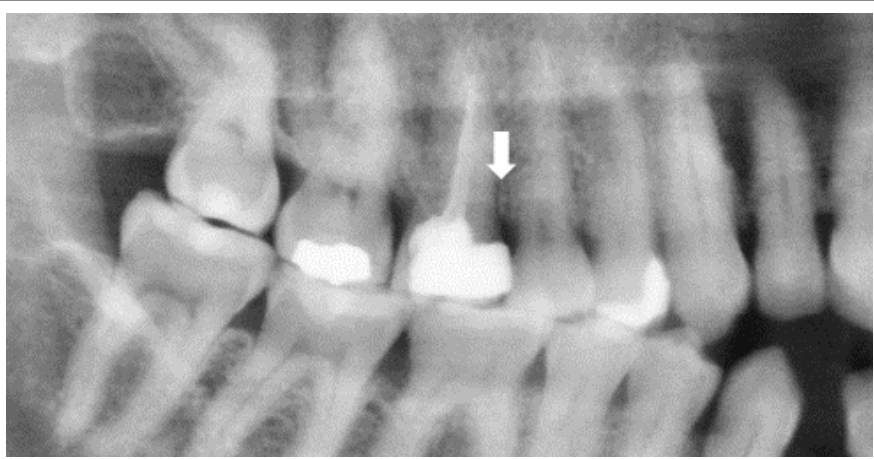

Figure 1: Non-diabetic patient with class II amalgam restoration; the age of restoration is 2 years, and the interproximal bone loss is indicated by an arrow between 16 and 17.

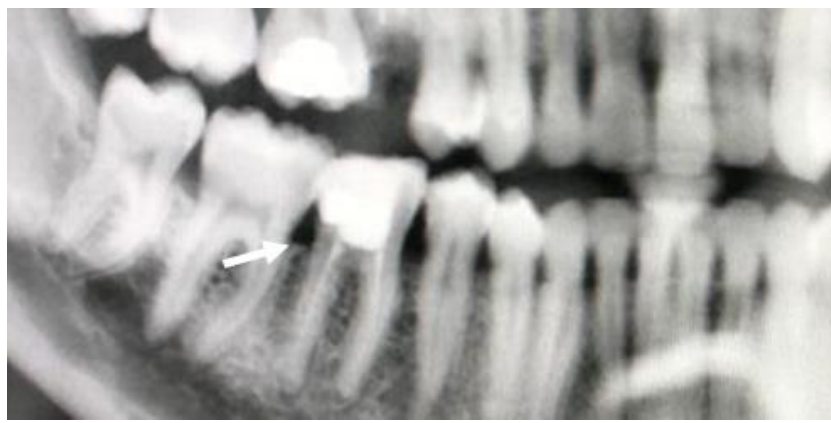

Figure 2: Non-diabetic patient with class II amalgam restoration; the age of restoration is 4 years, and the interproximal bone loss is indicated by an arrow between 46 and 47 .

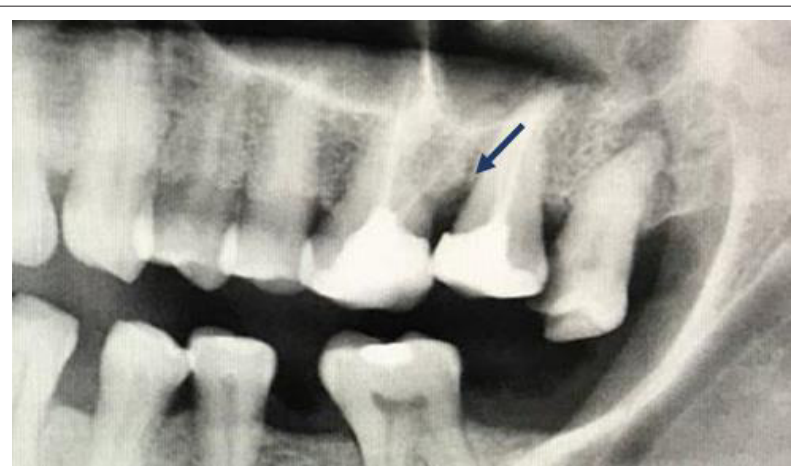

Figure 3: Non-diabetic patient with class II amalgam restoration; the age of restoration is 4 years, and the interproximal bone loss is indicated by an arrow between 46 and 47.

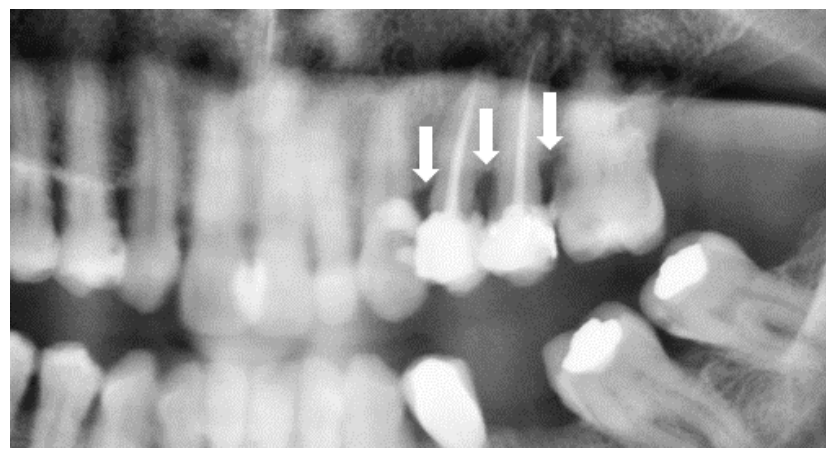

Figure 4: Areas of different amounts of alveolar bone loss, towards class II amalgam restorations; these areas are indicated by arrows in the interproximal areas: (1) 23 , and $24,(2) 24$ and 25 , and (3) 25 and 26. 


\section{Discussion}

The participants of the examined groups (G1-G5) demonstrated different mean percentages of ABL values, which ranged from $25 \%$ to $41 \%$, which suggested periodontal destructions, caused by different stages of chronic periodontitis. Nevertheless, G1 and G2 who had their class amalgam restorations for relatively short service lives ( 2 and 4 years, respectively), demonstrated mild ABL scores, indicative of mild chronic periodontitis. For 7, 10, and $>10$ years of service life of class II amalgam restorations (G3, G4, and G5), the ABL values escalated significantly, indicating further periodontal destructions. This steady increment in the ABL values with the time of the class II amalgam restorations suggested a directly proportional relationship between the service life of class II amalgam restorations and stage of periodontitis. These ABL values have been quantified in proximal areas (mesial or distal), towards the restored portion of the class II amalgam restorations. Hence, the amount of localized ABL might have essentially been affected by two factors; these are related to class II amalgam restorations: 1) class II amalgam overhangs and 2) cavity-restoration interface in the interproximal area. Owing to features of the two factors, further plaque accumulation, and increased progression of gingival inflammation are inevitable in such areas. Therefore, it is anticipated that these areas are more prone to periodontal destruction, and its subsequent complications, such as increased ABL.

When the PI and GI scores of G1-G5 were examined, there were considerable variations among these values. While G1-G3 had low to intermediate PI and GI scores, G4 and G5 demonstrated higher scores for the same parameters. The low to intermediate PI and GI scores of G1-G3 indicated that the participants of these groups had relatively better periodontal status than those of G4 and G5. An explanation for the poor periodontal parameters among participants of G4 and G5 was clearly depicted by clinical examination, where most of these participants showed poor oral hygiene, as indicated by high PI, and severe gingival inflammation as, indicated by high GI. Considering these periodontal parameters' values, and how they interrelate with the $A B L$ values showed that the mild to moderate ABL values of G1-G3 were as per the low to intermediate scores of PI and GI. Likewise, higher scores of the PI and GI of G4 and G5 were accompanied by higher ABL values. PI and GI are evaluated considering the entire status of the hard and soft tissues of the oral cavity. Therefore, the presence of dental restorations such as class II amalgam restoration should not be solely responsible for the general periodontal health status, unless for substantially or full mouth reconstruction, which is not the case of the present investigation. Except for the surrounding area, the whole effect of a dental restoration should not be extended beyond the restored area. Therefore, it can be speculated that PI and GI scores are independent of the effect of dental restorations as dental restoration can be responsible for the localized periodontal inflammation of the restored area, and its surroundings.

The prevalence of $\mathrm{OH}$ among the participants of the examined groups (G1-G5) ranged from $40 \%$ to $60 \%$. The highest incidence of overhanging restorations, found among participants of G1, was accompanied by the lowest amount of ABL. G2 which had similar ABL to that of G1 had significantly a lower prevalence of OH. G4 and G5 demonstrated the highest amount of ABL despite their lower rate of $\mathrm{OH}$ than all other groups. These results indicated that the overhang's incidence did not correlate with ABL, which might be attributed to the effect of size of the restorations and/or size of overhangs' margins. It is well established that overhang's margin less than $0.2 \mathrm{~mm}$ should be harmless to the periodontal health. It is noteworthy that ABL correlated significantly with the service life of these restorations, but not with the incidence of overhanging restorations. Therefore, it can be speculated that longer service life (age) of overhanging restoration is more detrimental to periodontal health, and subsequently further ABL than merely the prevalence of overhanging restorations.

Another risk factor for the periodontal complications among the examined groups was the age of participants. It is well-known that there is a significant association between age, as a systemic risk factor, and periodontal health $[35,36]$. Therefore, when the age group is interrelated to the resultant periodontal parameters, two outcomes are equally conceivable. Firstly, participants with older ages will present with worse periodontal parameters as compared to younger ones. Secondly, participants with similar age groups will be accompanied by similar periodontal parameters. In the current study, the participants of the G4 and G5, the oldest among the examined groups presented with the worst periodontal parameters. However, G1, G2, and G3, who had similar age groups, were shown to have different periodontal parameter values. Therefore, it is rational to speculate that periodontal outcomes are not necessarily stemming from the effect of a single risk factor, such as aging. Other risk factors should be relevant in this respect, such as oral hygiene status, the effect of dental restoration, and its related factors, such as service life, smoothness, overhang, and subgingival margin. Therefore, it is anticipated that the synergistic action of these risk factors is detrimental to periodontal health.

The significant association between periodontal health and dental restorations is well documented in the literature. A literature review presented several published data that showed the effect of restorative dental materials on the health and safety of gingival tissues and tooth surrounding structures. These studies showed variation in the degree of plaque accumulation among patients treated with different restorative materials. Alsanjary et al [28] stated that class II amalgam restorations were associated with relatively low PI (1.16) and GI (1.5); these values were like those 
of class II composite resins. In a cross-sectional study by Ababnaeh et al. [26], it has been reported that amalgam restorations were associated with the highest PI as compared with other dental restorations, such as composite resins. Al-Fawaz et al. [27] reported lower PI and GI scores of class II amalgam restorations $(1.57,1.24)$ as compared with those of class II composite resin restorations $(1.73,1.58)$. Another study by Al-Abdaly et al. [29] has reported that class II amalgam restorations had higher PI and GI scores than those of metal-ceramic crowns. Notably, these periodontal parameters' outcomes are different. One explanation for such differences might be the diverse clinical conditions, such as different service life of dental restorations, health status of participants, oral hygiene, and age. A recent cross-sectional study by the current investigator [37] evaluated the effect of service life of class II amalgam restorations on periodontal health of a sample of patients with diabetes. The outcomes of the periodontal parameters reported, namely, the ABL, PI, and GI scores, were remarkably higher than those of the current study. The previous periodontal research study [37] that reported higher periodontal scores than the current study examined patients with type-2 diabetes mellitus, a well-known systemic disease that is known to increase periodontal disease risk and its complications. Whereas the present study evaluated the periodontal indices of individuals with no diabetes. Therefore, it is anticipated that different outcomes might be obtained when comparing results under dissimilar clinical conditions.

There are some limitations of the current study which might have biased the outcomes of the present study. Women were not included in this study. Hormonal changes in women were found to have an impact on periodontal health [38]. The fact that men have a higher rate of periodontal disease is true [39]. Moreover, there is still a necessity for research studies that evaluate the severity of periodontal disease among men and women. Although the effect of obesity on periodontal health, as a risk factor for the increased susceptibility of periodontal complications is inevitable [40]; it was not considered in the present study. Smoking is another important limitation of the present study. It is a well-known risk factor for numerous medical conditions, including periodontal disease [41]. The limitations that are related to class II amalgam restorations that should not be overlooked include restorations' smoothness, size of restorations, and size of proximal overhangs. Further research studies that consider different aspects of these limitations are needed.

\section{Conclusion}

The examined participants demonstrated a wide range of values for the assessed periodontal parameters (ABL, PI, and GI). This emphasizes various features of the relationship that exists between service life of class II amalgam restoration, age of participants, overhanging restorations, oral hygiene status, and periodontal health. A high prevalence of overhanging restorations, as observed in some groups, does not always imply inferior periodontal measures, as was originally assumed. Other considerations should include the extent of the overhang's margin, the service life of class II amalgam restoration, and the participant's age. In particular, the worsening periodontal parameters may not be primarily due to a single risk factor. The synergistic action of manifold risk factors is anticipated to cause significant periodontal destruction and subsequent deterioration of periodontal parameters. However, since the service life of class II amalgam restorations and age of participants correlated consistently with the worse periodontal parameters in the current study, it can then be assumed that these risk factors were the most influential for the worse periodontal parameters among the examined participants.

\section{Acknowledgments}

The author is highly grateful to the faculty of dentistry, University of Najran, KSA, and would like to acknowledge the following people for their clinical assistance, Dr. Abdo Abdul Razzaq, Dr. Waleed Aseeri, and Professor Michael Vincent Swain for revising the manuscript.

\section{Funding}

The author declares no specific funding or other financial support for the present investigation.

\section{Author Contribution}

The author confirms sole responsibility for study conception and design, data collection, analysis and interpretation of results, and manuscript preparation.

\section{Conflict of Interest}

The author declares that the work documented in this scientific article is not recognized for any conflicting financial interests and/ or personal connections.

\section{References}

1. Könönen E, Gursoy M, Gursoy UK (2019) Periodontitis: A Multifaceted Disease of Tooth-Supporting Tissues. J Clin Med 31(8): 1135.

2. Armitage GC (2004) Periodontal diagnoses and classification of periodontal diseases. Periodontol 2000 34: 9-21.

3. Kim J, Amar S (2006) Periodontal disease and systemic conditions: a bidirectional relationship. Odontology 94(1): 10-21.

4. Pinto G, Silva MD, Peddey M, Sillankorva S, Azeredo J (2016) The role of bacteriophages in periodontal health and disease. Future Microbiol 11: 1359-1369.

5. Dahlen G, Basic A, Bylund J (2019) Importance of virulence factors for the persistence of oral bacteria in the inflamed gingival crevice and in the pathogenesis of periodontal disease. J Clin Med 29(8): 1399.

6. American Diabetes Association (2009) Diagnosis and classification of diabetes mellitus. Diabetes Care 32(1): 62-67. 
7. Abbass MMS, Rady D, Radwan, El Moshy S, AbuBakr N, et al. (2019) The occurrence of periodontal diseases and its correlation with different risk factors among a convenient sample of adult Egyptian population: a cross-sectional study. F1000Res 11 (8): 1740.

8. Nazir MA (2017) Prevalence of periodontal disease, its association with systemic diseases and prevention. Int J Health Sci (Qassim) 11(2): 72-80.

9. Bäumer A, Weber D, Staufer S, Pretzl B, Körner G, et al. (2020) Tooth loss in aggressive periodontitis: Results 25 years after active periodontal therapy in a private practice. J Clin Periodontol 47(2): 223-232

10. Armitage GC (1999) Development of a classification system for periodontal diseases and conditions. Ann Periodontol 4(1): 1-6.

11. AlJehani YA (2021) Risk factors of periodontal disease: review of the literature. Retraction in: Int J Dent 12 :8735071.

12. Bascones Martínez A, Figuero Ruiz E (2004) Periodontal diseases as bacterial infection. Med Oral Patol Oral Cir Bucal 9: 101-107.

13. Colombo APV, Tanner ACR (2019) The role of bacterial biofilms in dental caries and periodontal and peri-implant diseases: A historical perspective. J Dent Res 98(4): 373-385.

14. Loesche WJ, Grossman NS (2001) Periodontal disease as a specific, albeit chronic, infection: diagnosis and treatment. Clin Microbiol Rev 14(4): 727-752.

15. Murakami S, Mealey BL, Mariotti A, Chapple ILC (2018) Dental plaqueinduced gingival conditions. J Periodontol 89(1): s17-2s7.

16. Seneviratne CJ, Zhang CF, Samaranayake LP (2011) Dental plaque biofilm in oral health and disease. Chin J Dent Res 14(2): 87-94.

17. Dhingra K, Vandana KL (2011) Indices for measuring periodontitis: a literature review. Int Dent J 61(2): 76-84.

18. Paolantonio M, D’ercole S, Perinetti G, Tripodi D, Catamo G, et al. (2004) Clinical and microbiological effects of different restorative materials on the periodontal tissues adjacent to subgingival class $\mathrm{V}$ restorations. J Clin Periodontol 31(3): 200-207.

19. Reddy KV, Nirupama C, Reddy PK, Koppolu P, Alotaibi DH (2020) Effect of iatrogenic factors on periodontal health: An epidemiological study. Saudi Dent J 32(2): 80-85.

20. Sirajuddin S, Narasappa KM, Gundapaneni V, Chungkham S, Walikar AS (2015) Iatrogenic Damage to Periodontium by Restorative Treatment Procedures: An Overview. Open Dent J 26(9): 217-222.

21. Ilday NO, Celik N, Dilsiz A, Alp HH, Aydin T, et al. (2016) The effects of overhang amalgam restoration on levels of cytokines, gingival crevicular fluid volume and some periodontal parameters. Am J Dent 29(5): 266270.

22. Jeffcoat MK, Howell TH (1980) Alveolar bone destruction due to overhanging amalgam in periodontal disease. J Periodontol 51(10): 599-602.

23. Parsell DE, Streckfus CF, Stewart BM, Buchanan WT (1998) The effect of amalgam overhangs on alveolar bone height as a function of patient age and overhang width. Oper Dent 23(2): 94-99.

24. Kells BE, Linden GJ (1992) Overhanging amalgam restorations in young adults attending a periodontal department. J Dent 20(2): 85-89.
25. Najm AA, Akram HM, Mahdi AA, Ali OH (2018) Clinical and radiographical assessment of alveolar bone loss associated with overhang amalgam filling. Int J Med Res Health Sci 7(1): 11-16.

26. Ababnaeh KT, Al Omari M, Alawneh TN (2011) The effect of dental restoration type and material on periodontal health. Oral Health Prev Dent 9(4): 395-403.

27. Al Fawaz Y, Alofi R, Diab H (2017) Comparative study between the effect of class II amalgam and composite restorations in posterior teeth on periodontal tissues health. Egypt Dent J 63(3): 2571-2577.

28. AL Sanjary SA, Gasgoos SS (2017) Effect of class II amalgam and composite restorations on periodontal health of posterior teeth: an in vivo study. JODR 4(2): 82-92.

29. Al Abdaly M, Khawshal A, Alqisi, A, Al shari H, Alshahrani N, et al. (2018) Clinical and Radiographic Evaluation of Marginal Bone Loss and Periodontal Parameters after Various Dental Reconstruction Procedures. Int J Clin Med 9(1): 39-48

30. Al Sinaidi A, Preethanath RS (2014) The effect of fixed partial dentures on periodontal status of abutment teeth. Saudi J Dent Res 5(2): 104-108.

31. Crano W D, Brewer M B (2002) Principles and methods of social research (2nd ed) NJ: Lawrence Erlbaum Associates Publishers pp. 189-190.

32. Silness J, Löe H (1964) Periodontal disease in pregnancy. II. Correlation between oral hygiene and periodontal condition. Acta Odontol Scand 22: 121-135.

33. Löe H, Silness J (1963) Periodontal disease in pregnancy. I. prevalence and severity. Acta Odontol Scand 21: 533-551.

34. Zhao H, Li C, Lin L, Pan Y, Wang H, et al. (2015) Assessment of alveolar bone status in middle aged chinese (40-59 Years) with chronic periodontitis--using CBCT. PLoS One 10(10): e0139553.

35. Huttner EA, Machado DC, de Oliveira RB, Antunes AG, Hebling E (2009) Effects of human aging on periodontal tissues. Spec Care Dentist 29(4): 149-155.

36. López R, Smith PC, Göstemeyer G, Schwendicke F (2017) Ageing, dental caries and periodontal diseases. J Clin Periodontol 44(18): S145-S152.

37. Albakry M (2021) The Effect of the different service lives of class II amalgam restorations on periodontal health among type 2 diabetes patients. Int J Dent Oral Health 7(5): dx.

38. Jafri Z, Bhardwaj A, Sawai M, Sultan N (2015) Influence of female sex hormones on periodontium: A case series. J Nat Sci Biol Med 6(1): S146-S149.

39. Schulze A, Busse M (2016) Gender differences in periodontal status and 0 ral hygiene of non-diabetes and type 2 diabetes patients. Open Dent J 9(10): 287-297.

40. Keller A, Rohde JF, Raymond K, Heitmann BL (2015) Association between periodontal disease and overweight and obesity: a systematic review. J Periodontol 86(6): 766-776.

41. Haber J, Wattles J, Crowley M, Mandell R, Joshipura K, et al. (1993) Evidence for cigarette smoking as a major risk factor for periodontitis. J Periodontol 64(1): 16-23. 